\title{
Antitubercular Nucleosides that Inhibit Siderophore Biosynthesis: SAR of the Glycosyl Domain
}

\author{
Ravindranadh V. Somu ${ }^{a}$, Daniel Wilson ${ }^{a}$, Eric M. Bennett ${ }^{a}$, Helena Boshoff ${ }^{b}$, Laura \\ Celia $^{c}$, Brian Beck ${ }^{c}$, Clifton E. Barry III ${ }^{b}$, Courtney C. Aldrich ${ }^{a} *$
}

${ }^{a}$ Center for Drug Design, Academic Health Center, University of Minnesota, Minneapolis, Minnesota 55455, ${ }^{\mathrm{b}}$ Tuberculosis Research Section, National Institute of Allergy and Infectious Diseases, Rockville, Maryland 20852-1742, ${ }^{\mathrm{C}} \mathrm{ATCC}$

\section{Table of Contents.}

Figure S1: Purification of MbtA S3

Figure S2: Titration of MbtA with inhibitor 8 S3

Figure S3: Saturation curve of MbtA with ATP_ S4

Figure S4: Saturation curve of MbtA with salicylic acid S4

Dose response curves for the inhibitors

Figure S5: Dose response curve for inhibitor 9

Figure S6: Dose response curve for inhibitor $\mathbf{1 0}$ S5

Figure S7: Dose response curve for inhibitor 11 S5

Figure S8: Dose response curve for inhibitor 12 S6

Figure S9: Dose response curve for inhibitor $\mathbf{1 3}$ S6 S7

${ }^{1} \mathrm{H}$ and ${ }^{13} \mathrm{C}$ NMR spectra:

${ }^{1}$ H NMR spectrum of 8 S8

${ }^{13} \mathrm{C}$ NMR spectrum of 8 S9

${ }^{1} \mathrm{H}$ NMR spectrum of 9 S10

${ }^{13} \mathrm{C}$ NMR spectrum of 9 $\mathrm{S} 11$

${ }^{1} \mathrm{H}$ NMR spectrum of $\mathbf{1 0}$ S12

${ }^{13} \mathrm{C}$ NMR spectrum of $\mathbf{1 0}$ S13

${ }^{1} \mathrm{H}$ NMR spectrum of $\mathbf{1 1}$ S14

${ }^{13} \mathrm{C}$ NMR spectrum of 11 S15 
${ }^{1} \mathrm{H}$ NMR spectrum of $\mathbf{1 2}$

${ }^{13} \mathrm{C}$ NMR spectrum of $\mathbf{1 2}$

S16

${ }^{1} \mathrm{H}$ NMR spectrum of $\mathbf{1 3}$

S17

${ }^{13} \mathrm{C}$ NMR spectrum of $\mathbf{1 3}$

S18

S19

HPLC purity of the inhibitors

Methods for analysis

S20

Table S1. HPLC purity of the inhibitors

S21 


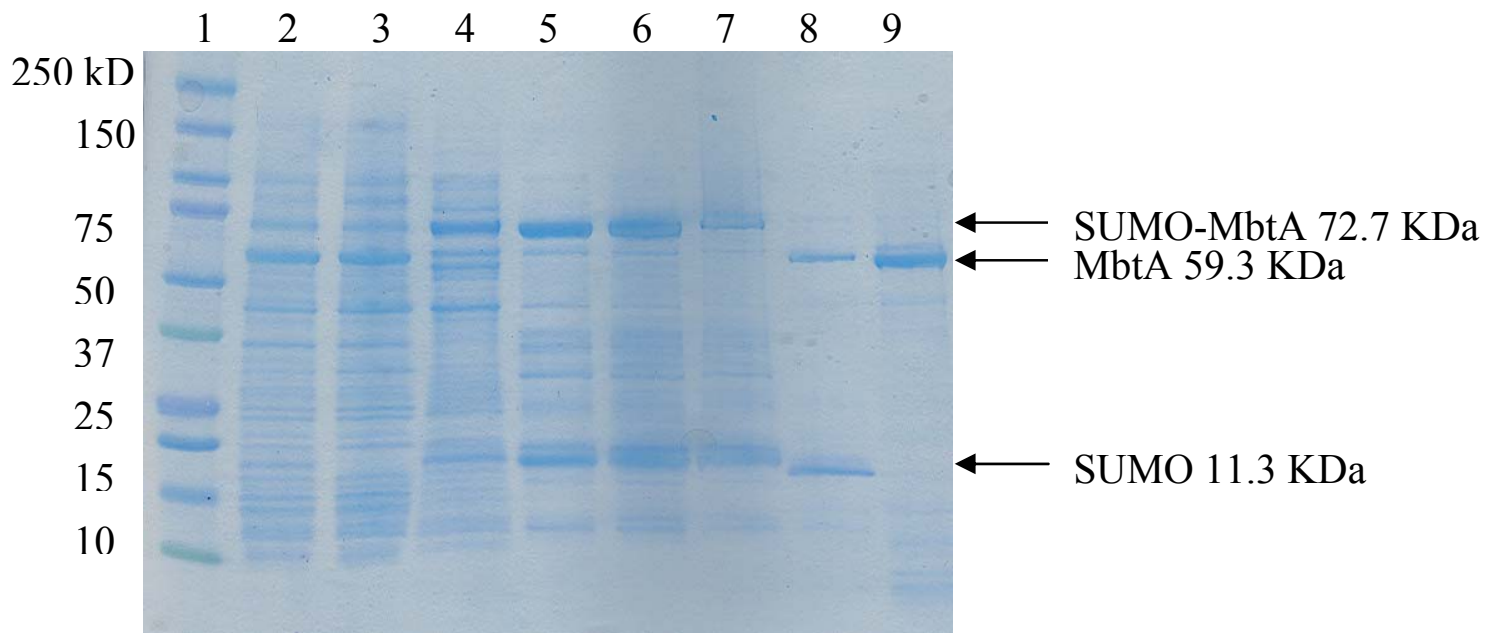

Figure S1: Purification of MbtA

1) molecular weight marker. 2) total soluble fraction. 3) Ni-NTA column flow through. 4) Ni-NTA column wash. 5) Ni-NTA column elution 6) PD-10 elution 7) PD-10 elution from fraction frozen overnight 8) SUMO protease digestion products 9) Purified MbtA



Figure S2: Titration of MbtA with analog 8 


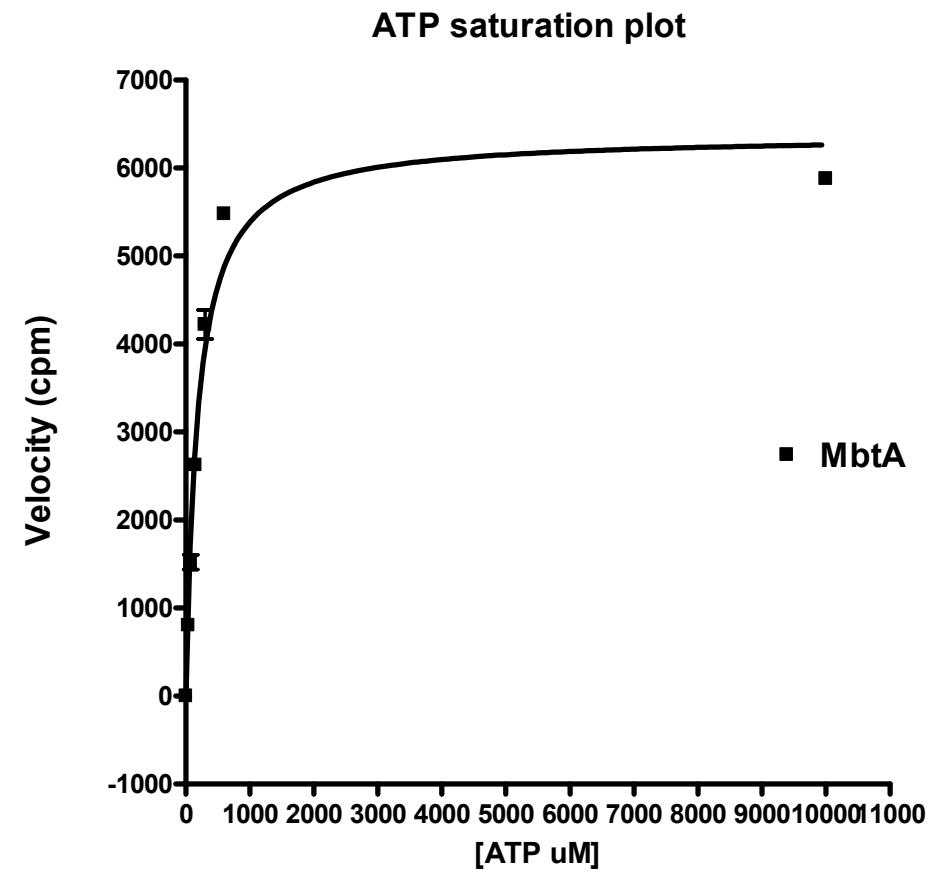

Figure S3: Saturation curve of MbtA with ATP

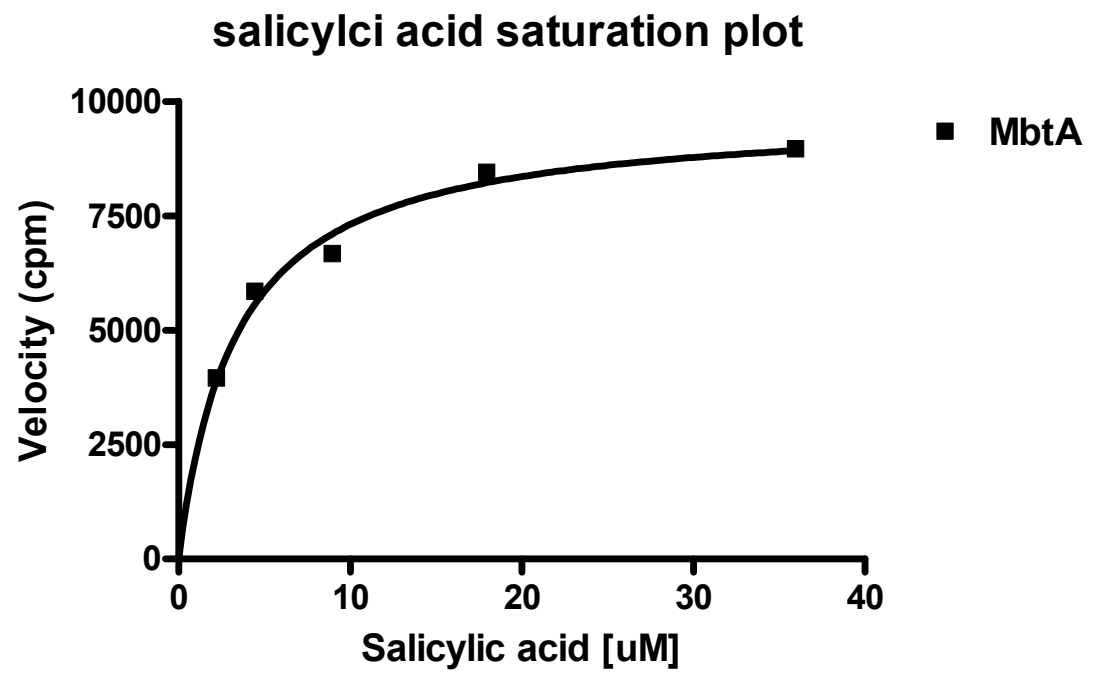

Figure S4: Saturation curve of MbtA with salicylic acid 


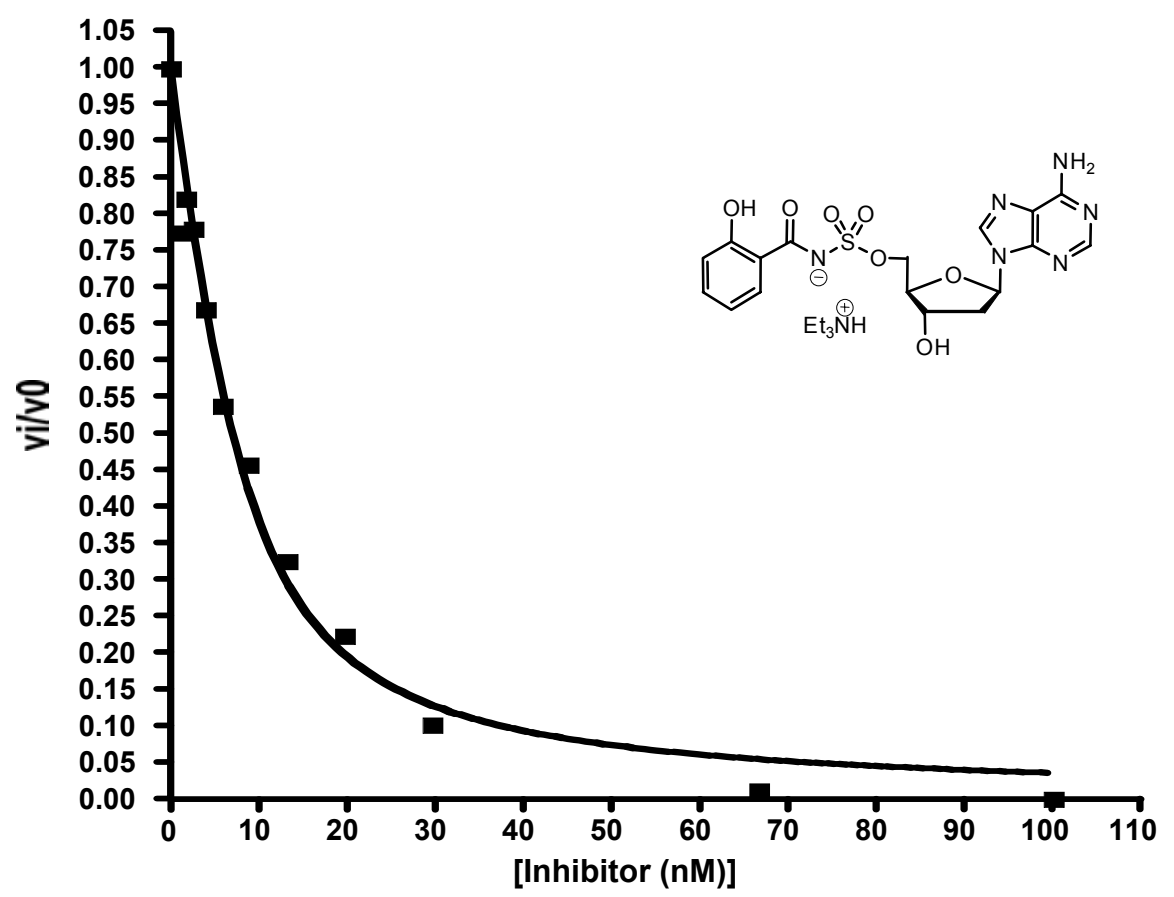

Figure S5: Dose response curve for inhibitor 9

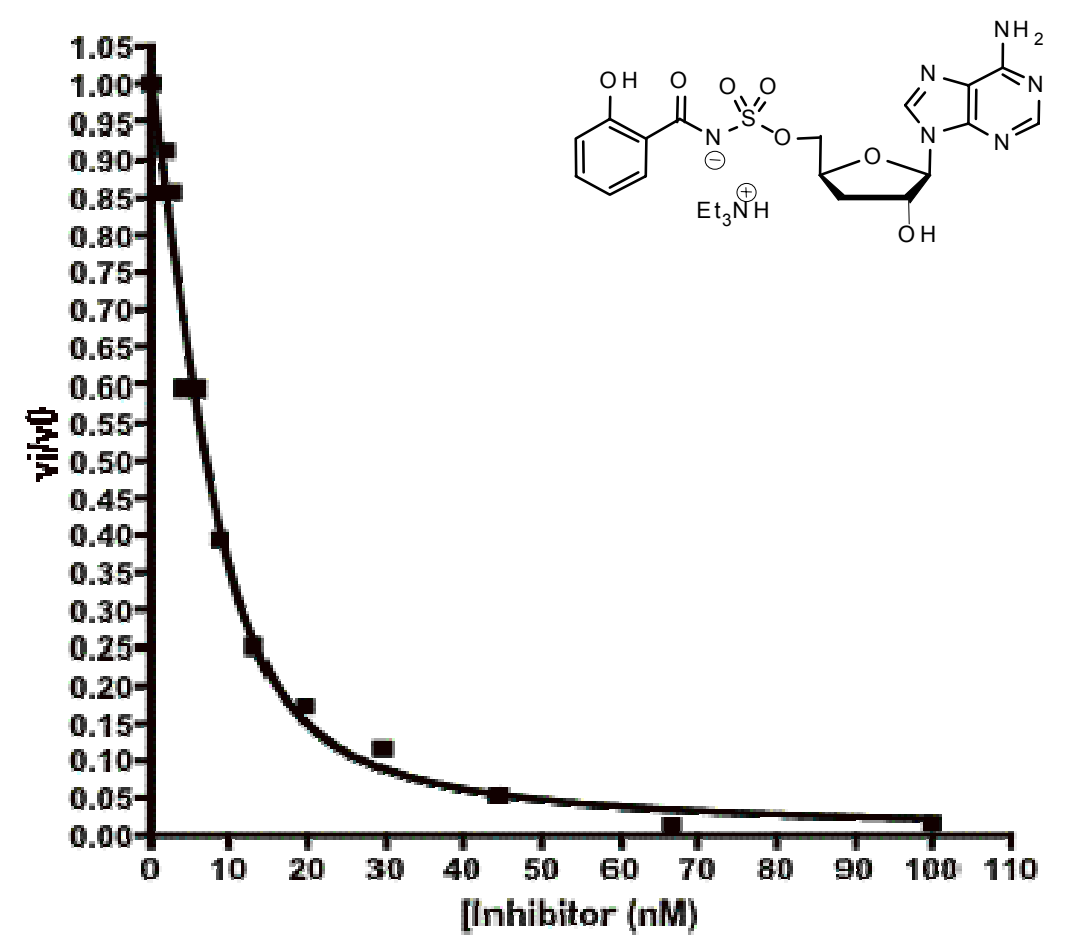

Figure S6: Dose response curve for inhibitor 10 


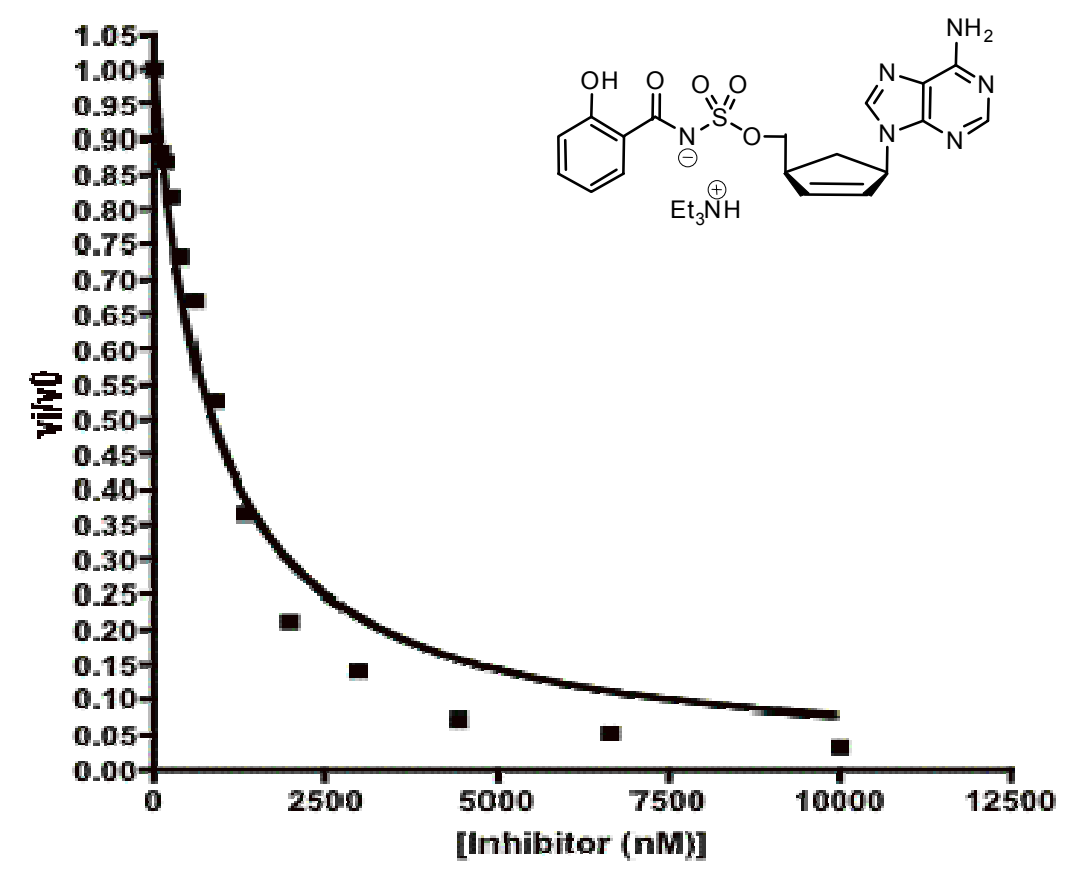

Figure S7: Dose response curve for inhibitor 11

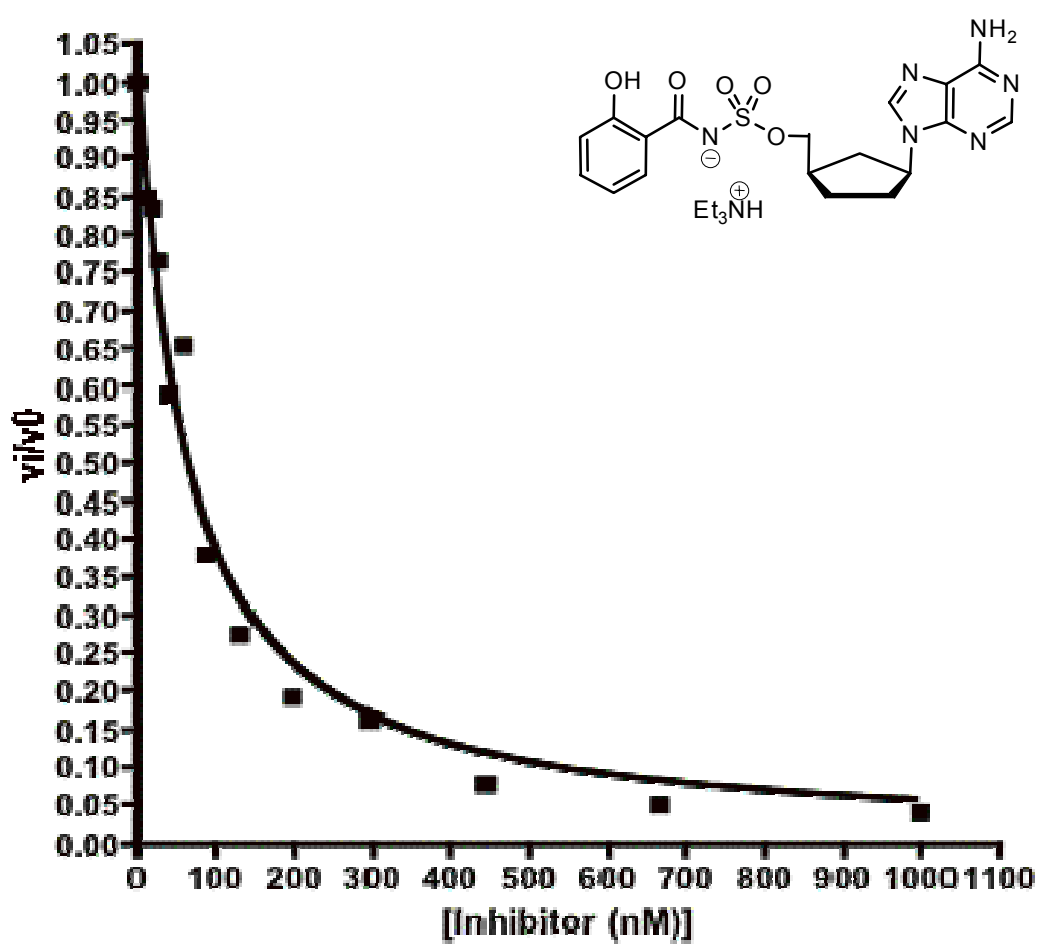

Figure S8: Dose response curve for inhibitor 12 


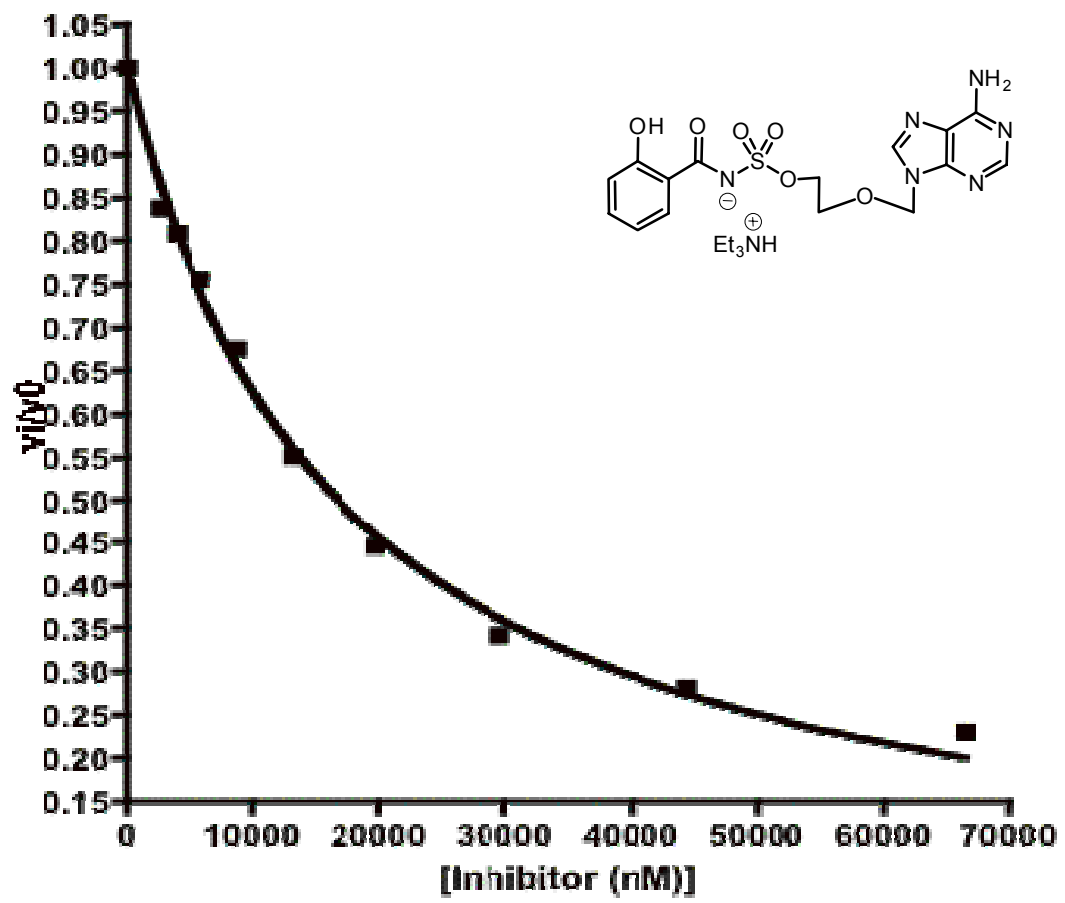

Figure S9: Dose response curve for inhibitor 12 


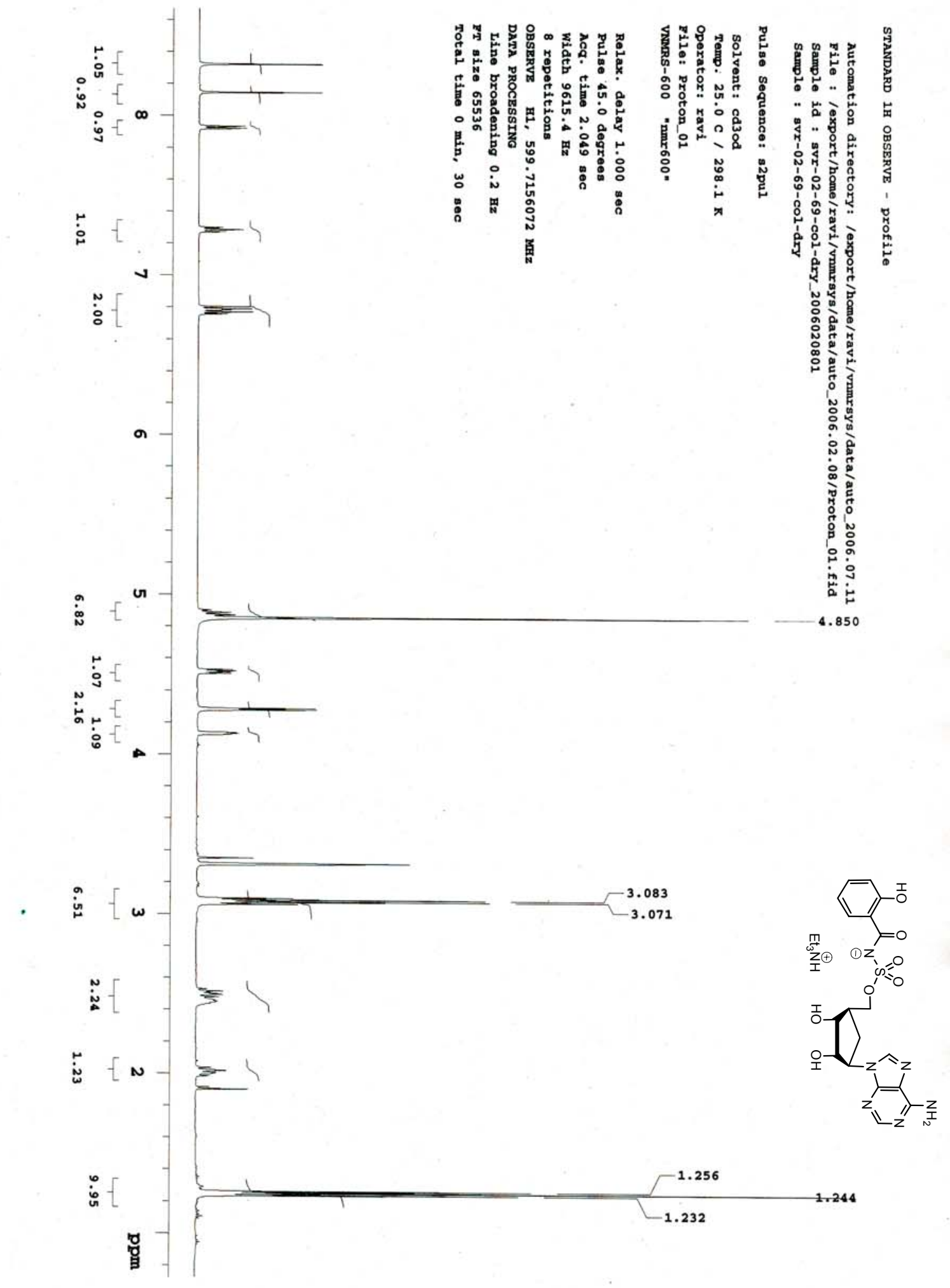




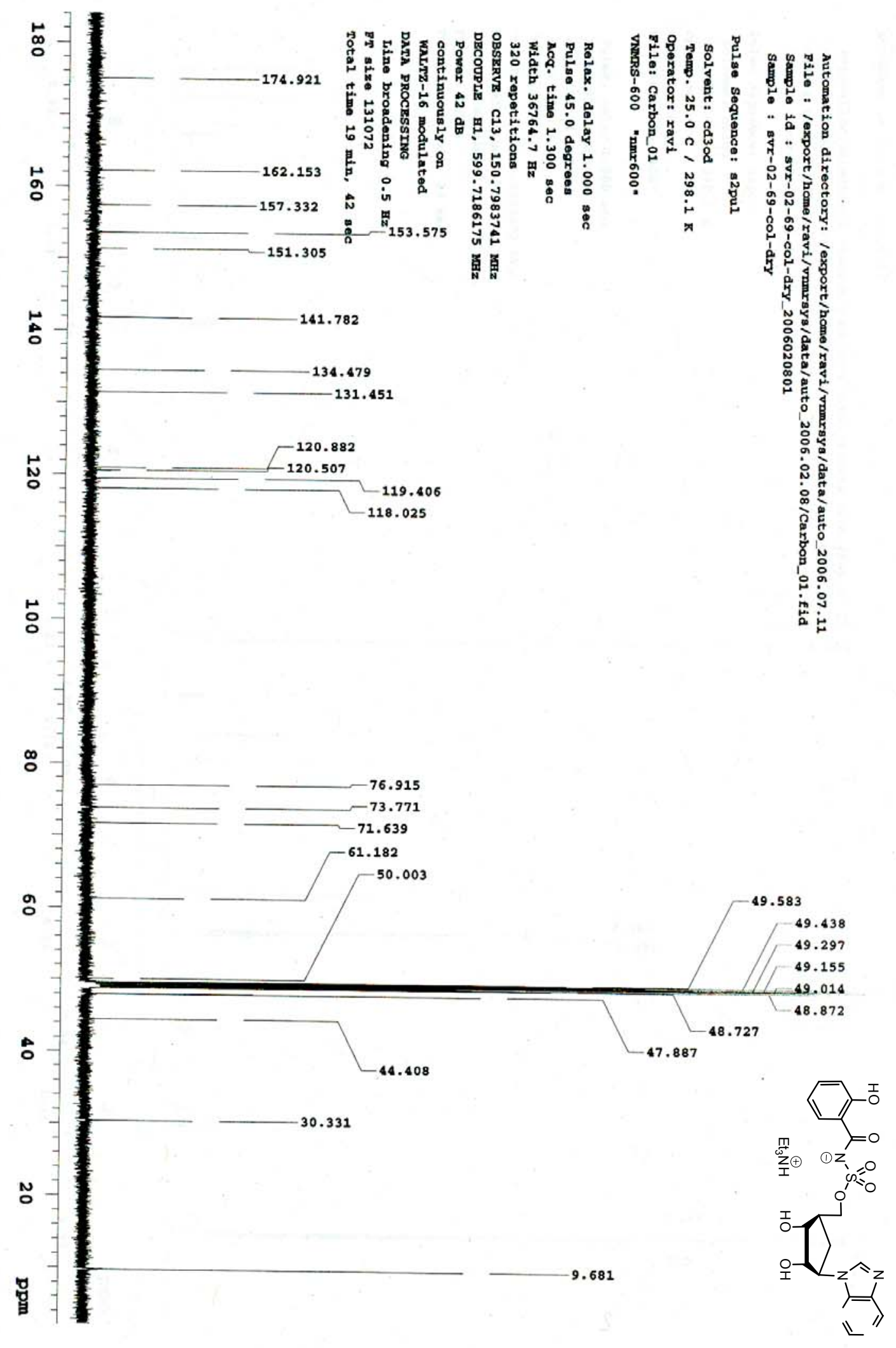









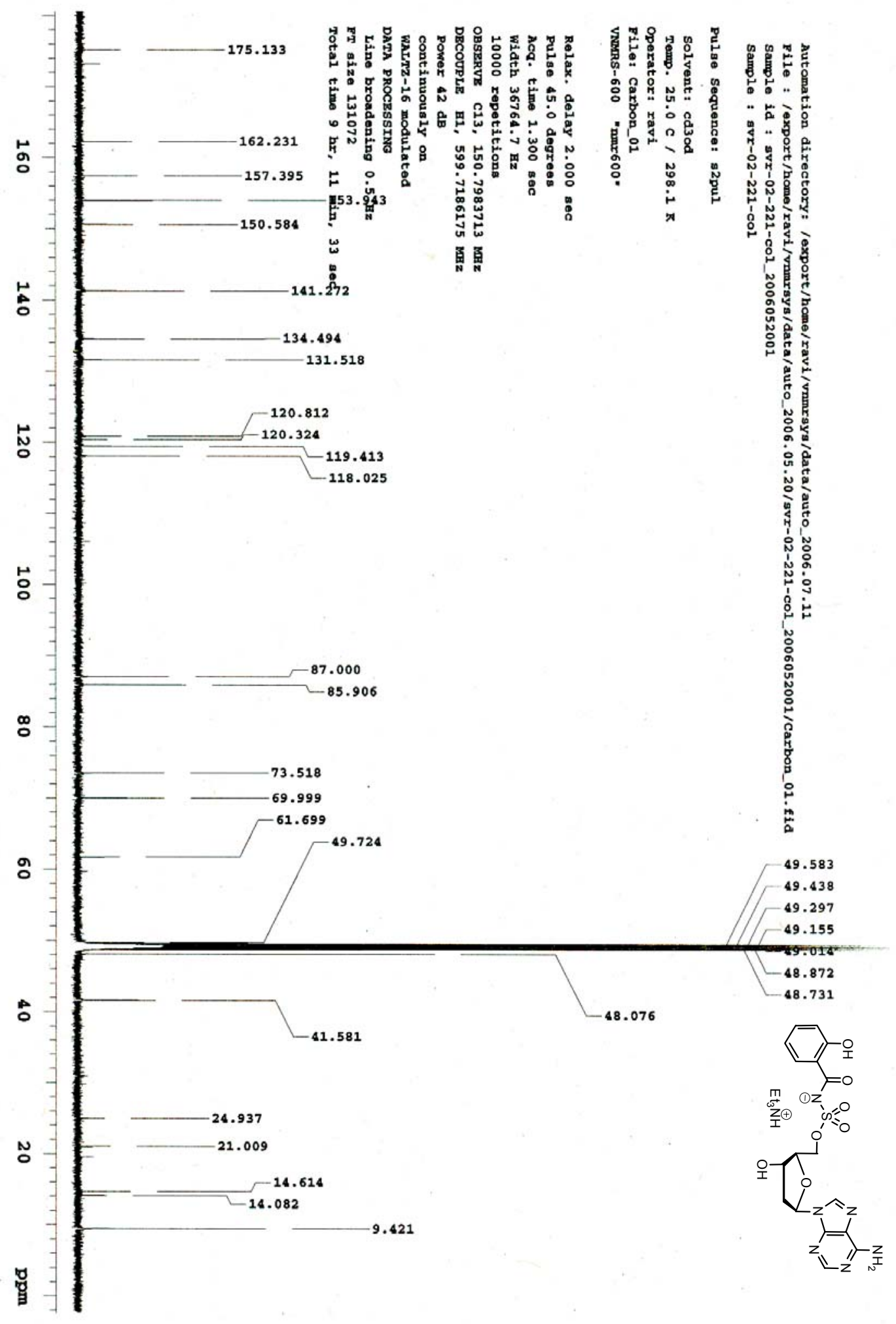




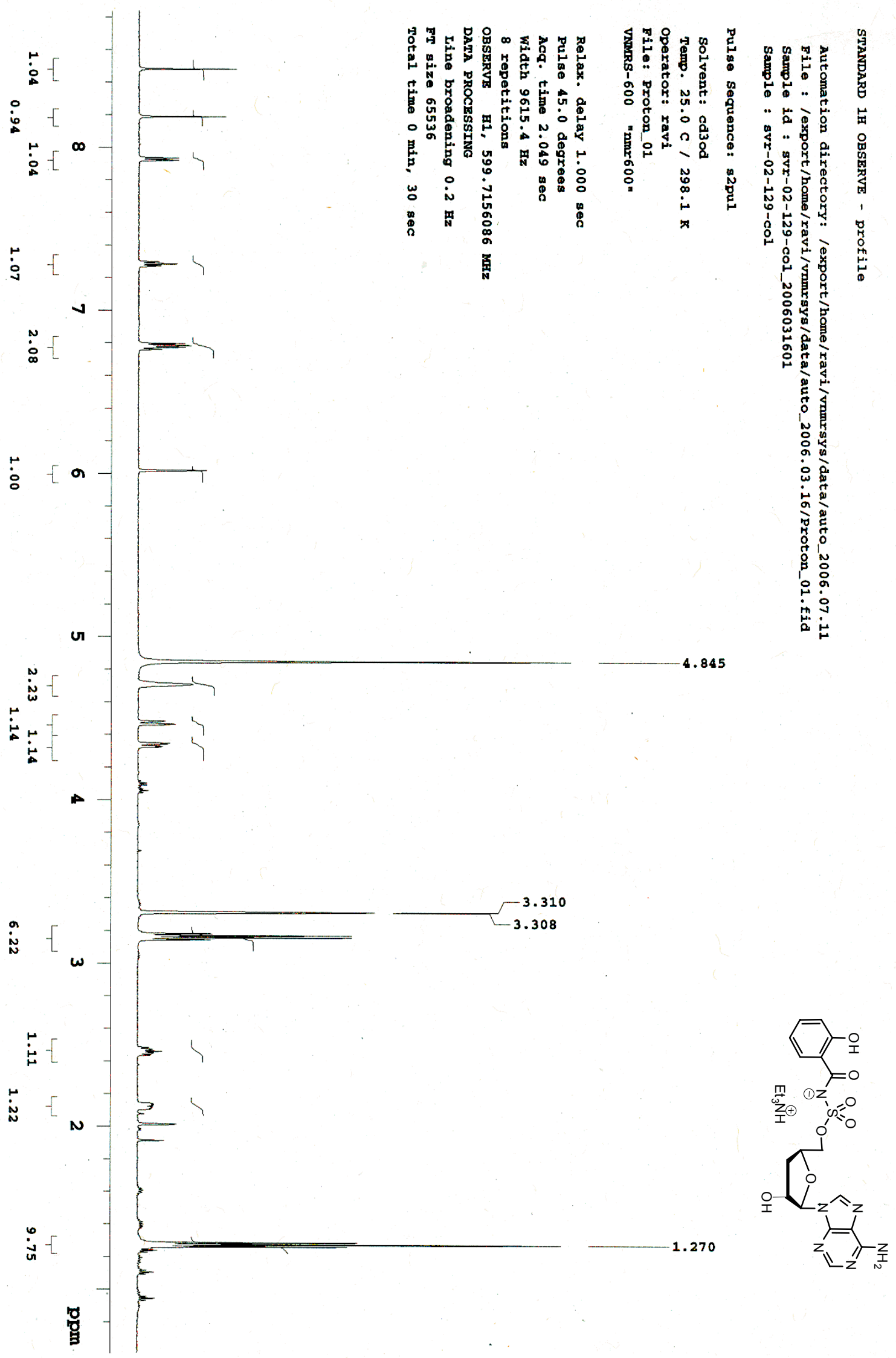




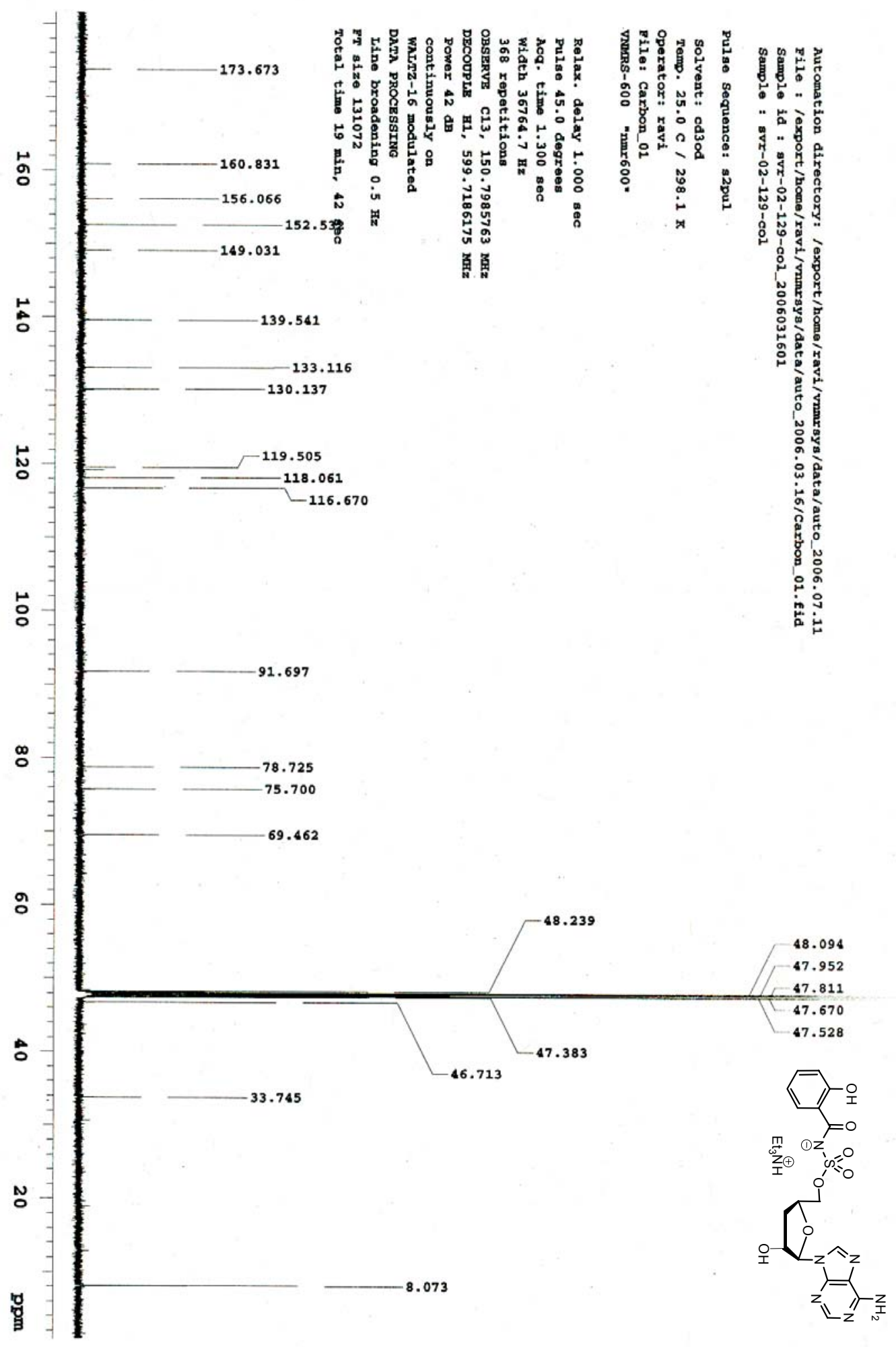









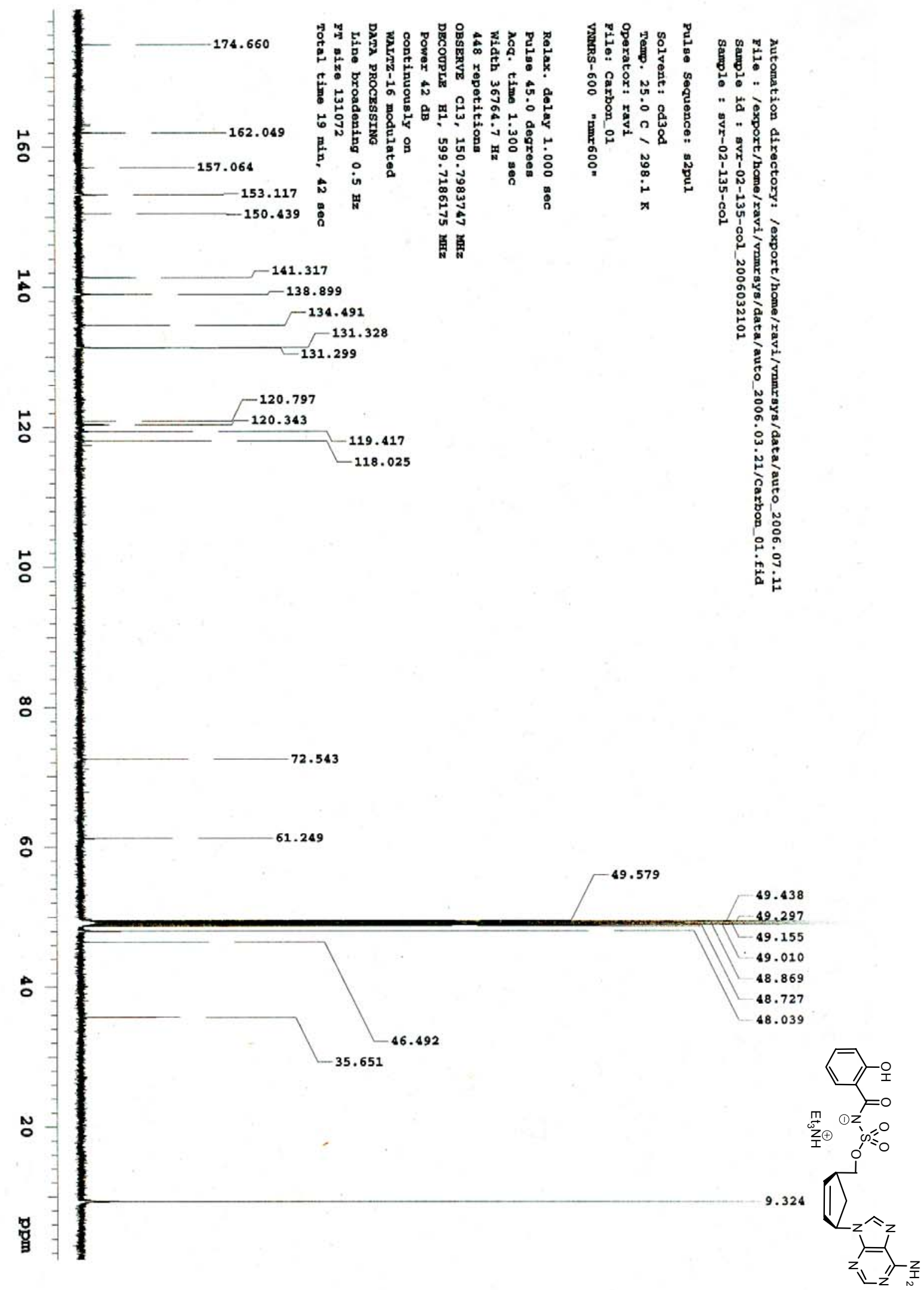




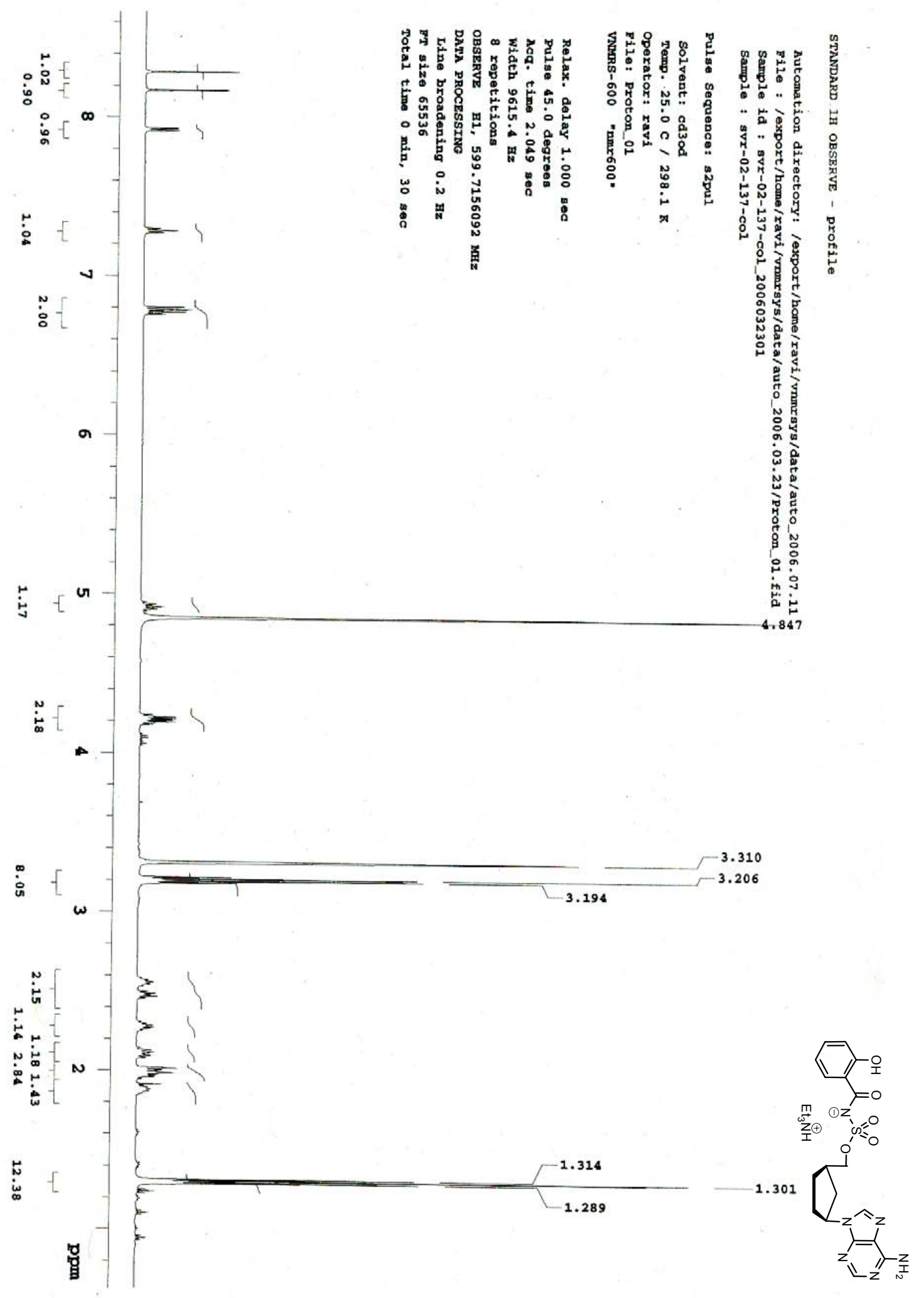




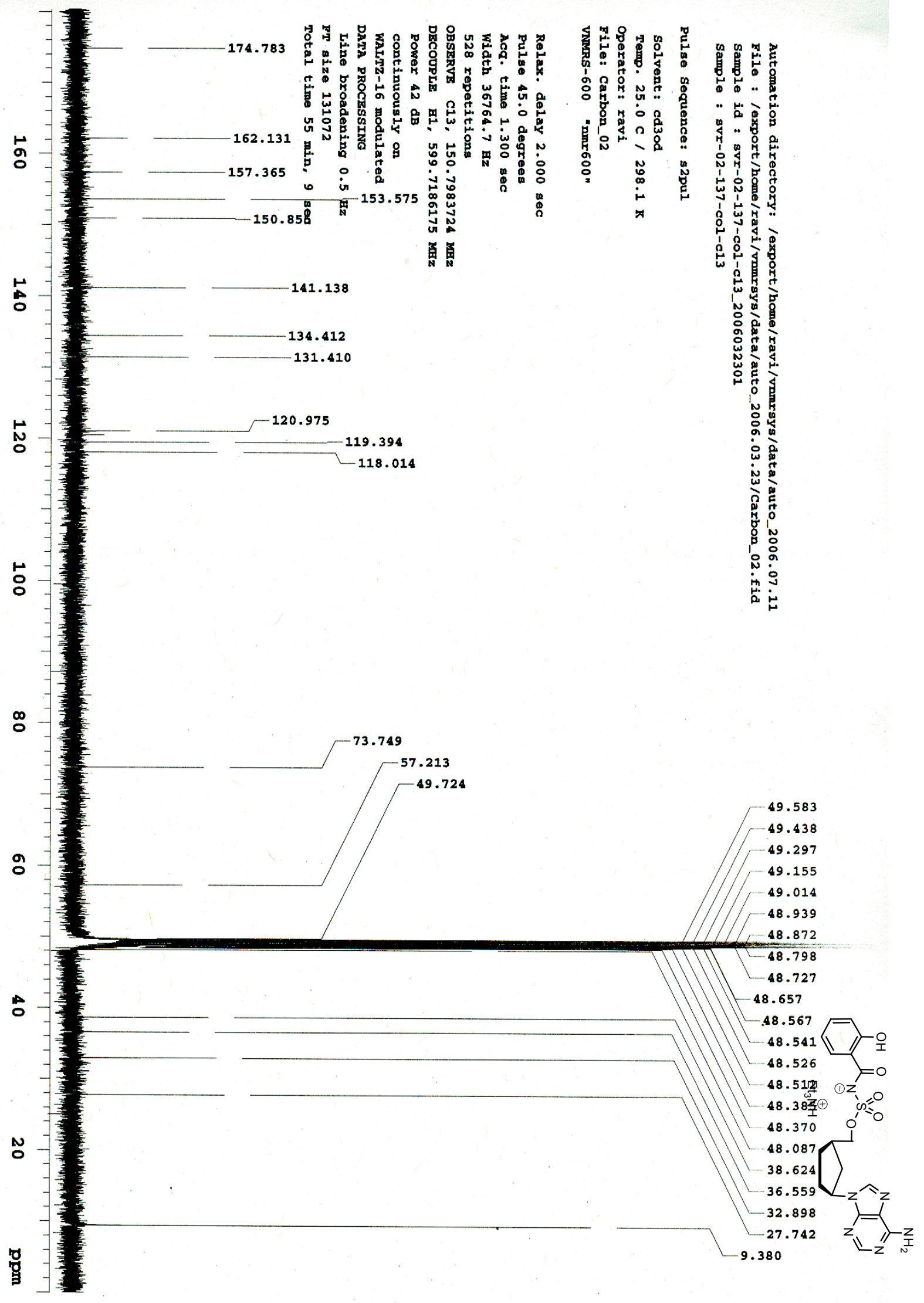




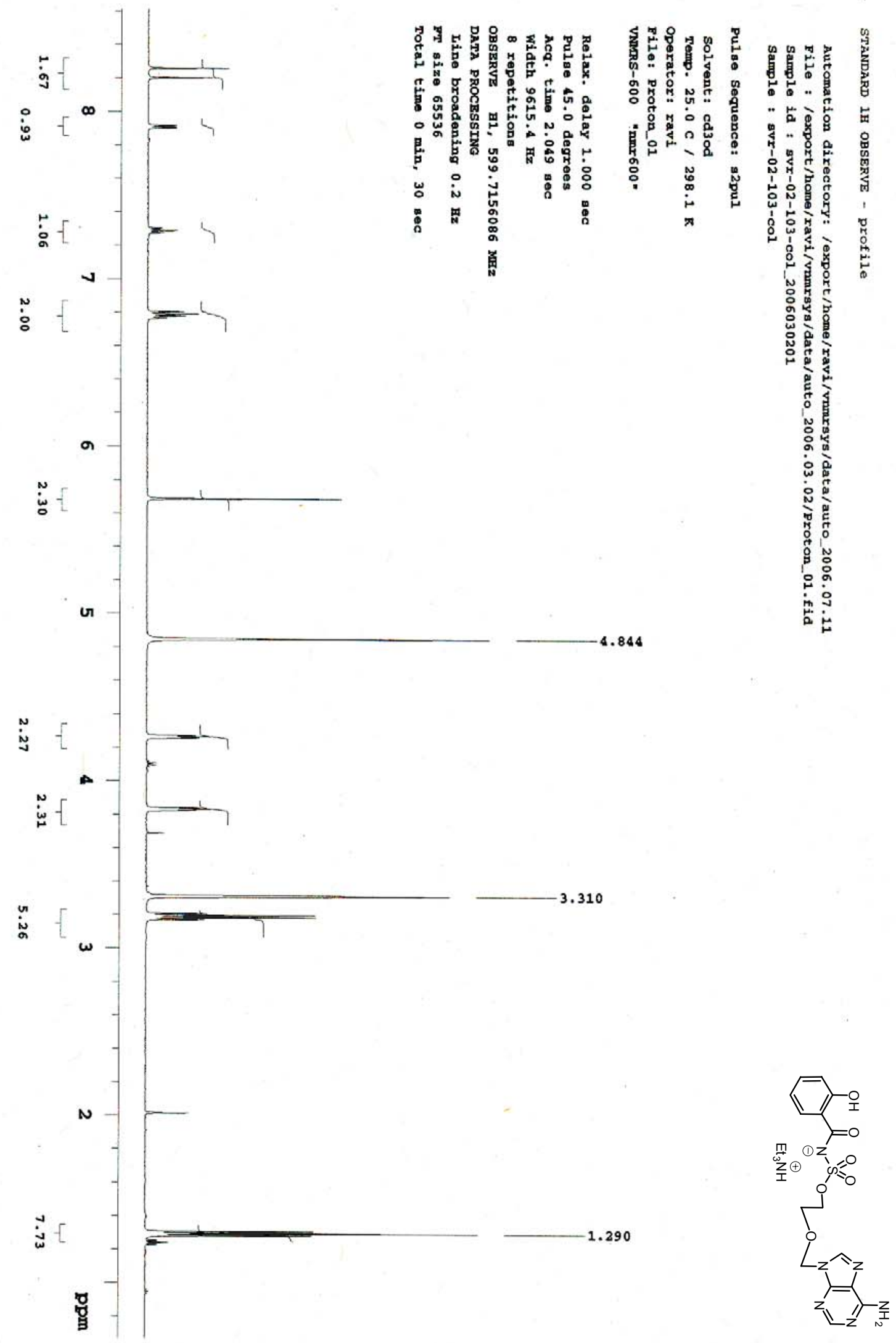




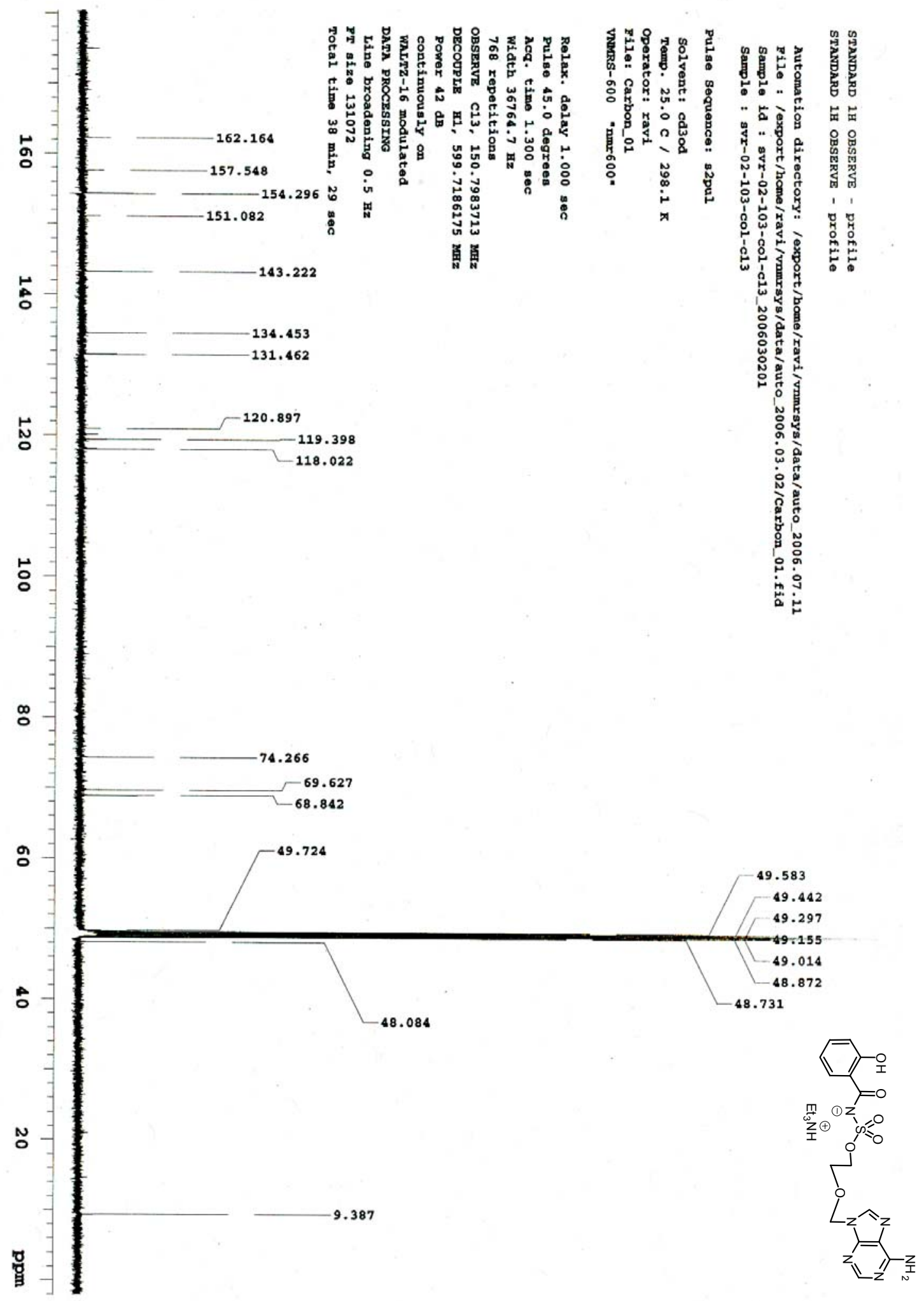


HPLC Purity of Inhibitors. HPLC purity was determined for all final target inhibitors 8-13. For all inhibitors two separate reverse-phase HPLC conditions were performed. An Agilent Eclipse XDB-C18 column $(4.6 \times 150 \mathrm{~mm}, 5 \mu \mathrm{m}$ particle size $)(\mathrm{Col} 1)$ or Varian Microsorb MV 100-5 C18 column (4.6 X $250 \mathrm{~mm}, 5 \mu \mathrm{m}$ particle size) (Col 2) with detection at $254 \mathrm{~nm}$ and the indicated HPLC conditions (Methods A-L) as described below was employed to determine the purity of inhibitors.

Method A: 0-20 min: gradient 10\%-100\% B; isocratic 20-25 min: $100 \% \mathrm{~B}$; gradient $25-$ 27 min: $100 \%-10 \%$ B; A: $0.04 \mathrm{M}$ triethyammonium bicarbonate; B: $70 \% \mathrm{CH}_{3} \mathrm{CN}-\mathrm{H}_{2} \mathrm{O}$, flow rate $0.5 \mathrm{ml} / \mathrm{min}$, Col 2 .

Method B: 0-14 min: isocratic 30\% B; gradient $14-15 \mathrm{~min}$ : $30-100 \% \mathrm{~B}$; isocratic $15-22$ min: $100 \%$ B; A: $0.05 \mathrm{M}$ triethyammonium bicarbonate; B: $70 \% \mathrm{CH}_{3} \mathrm{CN}-\mathrm{H}_{2} \mathrm{O}$, flow rate $0.5 \mathrm{ml} / \mathrm{min}, \mathrm{Col} 2$.

Method C: 0-20 min: gradient 0\%-90\% B; isocratic $20-27 \mathrm{~min}: 90 \% \mathrm{~B}$. A: $0.01 \mathrm{M}$ ammonium formate; $\mathrm{B}: 90 \% \mathrm{CH}_{3} \mathrm{CN}-\mathrm{H}_{2} \mathrm{O}$, flow rate $0.8 \mathrm{ml} / \mathrm{min}$, $\mathrm{Col} 1$.

Method D: 0-18 min: isocratic $25 \% \mathrm{~B}$; gradient $18-20 \mathrm{~min}$ : $25-100 \% \mathrm{~B}$; isocratic $20-23$ min: $100 \% \mathrm{~B}$, A: $0.01 \mathrm{M}$ ammonium formate; $\mathrm{B}: 90 \% \mathrm{CH}_{3} \mathrm{CN}-\mathrm{H}_{2} \mathrm{O}$, flow rate 0.8 $\mathrm{ml} / \mathrm{min}$, Col 1 .

Method E: 0-20 min: gradient 10\%-100\% B; isocratic 20-25 min: $100 \% \mathrm{~B}$; gradient $25-$ 27 min: $100 \%-10 \%$ B; A: $0.05 \mathrm{M}$ ammonium formate; $\mathrm{B}: 70 \% \mathrm{CH}_{3} \mathrm{CN}-\mathrm{H}_{2} \mathrm{O}$, flow rate $0.5 \mathrm{ml} / \mathrm{min}, \mathrm{Col} 2$.

Method F: 0-30 min: gradient 10\%-100\% B; isocratic 30-35 min: $100 \% \mathrm{~B}$; gradient 3537 min: $100 \%-10 \%$ B; A: $0.04 \mathrm{M}$ triethyammonium bicarbonate; B: $70 \% \mathrm{CH}_{3} \mathrm{CN}-\mathrm{H}_{2} \mathrm{O}$, flow rate $0.5 \mathrm{ml} / \mathrm{min}$, Col 2 .

Method G: 0-30 min: gradient 10\%-100\% B; isocratic 30-35 min: 100\% B; gradient 3537 min: $100 \%-10 \%$ B; A: $0.04 \mathrm{M}$ triethyammonium bicarbonate; B: $70 \% \mathrm{CH}_{3} \mathrm{CN}-\mathrm{H}_{2} \mathrm{O}$, flow rate $0.5 \mathrm{ml} / \mathrm{min}, \mathrm{Col} 2$.

Method H: 0-20 min: gradient 10\%-100\% B; isocratic 20-25 min: $100 \% \mathrm{~B}$; gradient $25-$ 27 min: $100 \%-10 \%$ B; A: $0.04 \mathrm{M}$ triethyammonium bicarbonate; B: $70 \% \mathrm{CH}_{3} \mathrm{CN}-\mathrm{H}_{2} \mathrm{O}$, flow rate $0.5 \mathrm{ml} / \mathrm{min}, \mathrm{Col} 2$.

Method I: 0-20 min: gradient 10-100\% B; isocratic 20-22 min: 100\% B; gradient 22-24 min: $100-10 \% \mathrm{~B}$; isocratic $24-30 \mathrm{~min}: 100 \% \mathrm{~B}, \mathrm{~A}: 0.04 \mathrm{M}$ triethyammonium bicarbonate; $\mathrm{B}: 70 \% \mathrm{CH}_{3} \mathrm{CN}-\mathrm{H}_{2} \mathrm{O}$, flow rate $0.5 \mathrm{ml} / \mathrm{min}, \mathrm{Col} 2$.

Method J: 0-30 min: isocratic 55\% B; 30-35 min: gradient $55-100 \% \mathrm{~B} ; 30-40 \mathrm{~min}$ : gradient $100-55 \%$ B. A: $0.04 \mathrm{M}$ triethyammonium bicarbonate; B: $70 \% \mathrm{CH}_{3} \mathrm{CN}-\mathrm{H}_{2} \mathrm{O}$, flow rate $0.5 \mathrm{ml} / \mathrm{min}, \mathrm{Col} 2$. 
Table S1. HPLC Purity of Inhibitors

\begin{tabular}{cccc}
\hline Inhibitor & Method & Retention time & Purity \\
& & & \\
\hline 8 & $\mathrm{~A}$ & 11.7 & $99 \%$ \\
\cline { 2 - 4 } & $\mathrm{B}$ & 11.2 & $97 \%$ \\
\hline \multirow{2}{*}{9} & $\mathrm{C}$ & 9.3 & $96 \%$ \\
\hline \multirow{2yyy}{*}{10} & $\mathrm{D}$ & 3.5 & $96 \%$ \\
\cline { 2 - 4 } & $\mathrm{E}$ & 17.3 & $96 \%$ \\
\hline 11 & $\mathrm{~F}$ & 20.1 & $94 \%$ \\
\hline 12 & $\mathrm{G}$ & 21.3 & $80 \%$ \\
\hline 13 & $\mathrm{H}$ & 18.5 & $82 \%$ \\
\cline { 2 - 4 } & $\mathrm{I}$ & 17.5 & $98 \%$ \\
\hline
\end{tabular}

